\title{
p53 GENE MUTATION AS BIOMARKER OF RADIATION INDUCED CELL INJURY AND GENOMIC INSTABILITY
}

\author{
Mukh Syaifudin \\ Center for Radiation Safety and Metrology Technology, NNEA \\ Jl. Cinere Pasar Jum'at PO BOX 7043 JKSKL Jakarta 12700
}

\begin{abstract}
p53 GENE MUTATION AS BIOMARKER OF RADIATION INDUCED CELL INJURY AND GENOMIC INSTABILITY. Gene expression profiling and its mutation has become one of the most widely used approaches to identify genes and their functions in the context of identify and categorize genes to be used as radiation effect markers including cell and tissue sensitivities. Ionizing radiation produces genetic damage and changes in gene expression that may lead to cancer due to specific protein that controlling cell proliferation altered the function, its expression or both. P53 protein encoded by $p 53$ gene plays an important role in protecting cell by inducing growth arrest and or cell suicide (apoptosis) after deoxyribonucleic acid (DNA) damage induced by mutagen such as ionizing radiation. The mutant and thereby dysfunctional of this gene was found in more than $50 \%$ of various human cancers, but it is as yet unclear how p53 mutations lead to neoplastic development. Wild-type $p 53$ has been postulated to play a role in DNA repair, suggesting that expression of mutant forms of $p 53$ might alter cellular resistance to the DNA damage caused by radiation. Moreover, $p 53$ is thought to function as a cell cycle checkpoint after irradiation, also suggesting that mutant $p 53$ might change the cellular proliferative response to radiation. $p 53$ mutations affect the cellular response to DNA damage, either by increasing DNA repair processes or, possibly, by increasing cellular tolerance to DNA damage. The association of $p 53$ mutations with increased radioresistance suggests that alterations in the $p 53$ gene might lead to oncogenic transformation. Current attractive model of carcinogenesis also showed that $p 53$ gene is the major target of radiation. The majority of $p 53$ mutations found so far is single base pair changes (point mutations), which result in amino acid substitutions or truncated forms of the P53 protein, and are widely distributed throughout the evolutionarily conserved regions of the gene. Examination of p53 mutations in human cancer also shows an association between particular carcinogens and characteristic patterns of these mutations. Even among cancers of the same organ, the mutational spectrum of the $p 53$ gene could present different patterns by histologic subtype or risk factors and mutagen involved.
\end{abstract}

Keywords: p53, tumor suppressor gene, mutation, apoptosis, growth arrest, ionizing radiation

\section{INTRODUCTION}

All organisms consist of cells that multiply through cell division. An adult human being has approximately 100000 billion cells, all originating from a single cell, the fertilized egg cell. In adults there is also an enormous number of continuously dividing cells replacing those dying. Before a cell can divide it has to grow in size, duplicate its chromosomes and separate the chromosomes for exact distribution between the two daughter cells. These different processes are coordinated in the cell cycle that is controlled by a number of genes that forming a network where the alteration of function in one of these genes will affect to all of the system. Normal cells have a 
number of intrinsic mechanisms that involve molecular "gatekeeper" in order to protect them from rapid uncontrolled proliferation $[1,2]$.

For all living eukaryotic organisms it is essential that the different phases of the cell cycle are precisely coordinated. The phases must follow in correct order, and one phase must be completed before the next phase can begin. Errors in this coordination may lead to chromosomal alterations. Chromosomes or parts of chromosomes may be lost, rearranged or distributed unequally between the two daughter cells. This type of chromosome alteration is often seen in cancer cells [3]. Many studies have revealed that human cancers are caused by the progressive accumulation of activating mutations in oncogenes and loss of function mutations in tumor suppressor genes. A subset of tumour suppressor genes function to prevent tumor formation or growth by inducing cell cycle arrest or apoptosis in response to potentially oncogenic events such as after ionizing radiation exposures. For example, tumor suppressor genes such as $p 53$ and $R b$ encode proteins that are activated and restrain proliferation following expression of activated alleles of $\mathrm{H}$-ras oncogenes [4,5].

Numerous natural and men-made agents may interact with the biological materials such as human tissues, causing reversible deviations from homeostatic equilibrium or irreversible change. Death of cells resulting from radiation damage is probabilistic. In this case, important end-points like cancer will only result from secondary events, such as initiation and promotion occurring during regenerative cell proliferation induced by cytolethality. The major effects of ionizing radiation are on DNA, compromising cell survival, cell proliferation and proper physiological cell functioning. Only a small fraction of the radiation-induced molecular modifications occur in the DNA of the cell nucleus, but practically all experimental and theoretical evidence indicates that DNA, the major information carrier in living matter, is the critical target. To protect the integrity of the genetic information, most of the cellular DNA is present in multiple copies, and most cells have highly intricate enzyme systems to repair efficiently and effectively DNA damage based on information contained within undamaged strands. Such damage may lead to reproductive cell death, mutations in germ cells with possible deleterious effects in offspring, or to modifications in somatic cells enhancing the risk of cancer [6]. These genetic changes are key events in carcinogenesis.

There is growing acknowledgment of the usefulness of determining expression patterns to identify and categorize genes to use as biological markers. Cell and tissue sensitivity to ionizing radiation is related to many endogenous gene expression patterns. It is well known that various stimuli such as ionizing radiation produce genetic damage and changes in gene expression. This paper describes a biomarker or biological endpoints such as cellular and molecular changes in $p 53$ gene that can be used to indicate an exposure to IR, representing an early event that occurs as a result of IR 
interaction with living tissues, completed with conservatively observation on its function and mechanism of the $p 53$ gene encoding P53 protein that is vital for controlling cancer throughout the body.

\section{p53 TUMOR SUPPRESSOR GENE}

P53 or known as tumor protein 53 (TP53) was identified in 1979 by Arnold Levine from Princeton University, David Lane in Imperial Cancer Research Fund (UK), and Lloyd Old from Sloan-Kettering Memorial Hospital. It had been hypothesized to exist before as the target of the SV40 virus, a strain that induced development of tumors. The $p 53$ gene was first cloned in 1983 by Moshe Oren from Weizmann Institute. It was initially presumed to be an oncogene due to the use of mutated cDNA following purification of tumour cell mRNA. Its character as a tumor suppressor gene was finally revealed in 1989. In 1993, P53 was voted molecule of the year by Science magazine. This gene encompasses $20 \mathrm{~kb}$ of DNA; 11 exons (the first is non-coding) and $3.0 \mathrm{~kb}$ mRNA with $1179 \mathrm{bp}$ open reading frame [7].

P53 is a transcription factor that regulates the cell cycle and hence functions as a tumor suppressor. It is very important for cells in multicellular organisms to suppress cancer. P53 has been described as "the guardian of the genome", referring to its role in conserving stability by preventing genome mutation and instability [8]. The name is due to its molecular mass as a 53 kilodalton $(\mathrm{kDa})$ protein on SDS-PAGE. Official protein name is cellular tumor antigen P53 and its synonyms are tumor suppressor P53 or phosphoprotein P53, or antigen NY-CO-13. The human $p 53$ gene is located on the human chromosome 17 (17p13.1). The location has also been mapped on other model animals: mouse (chromosome number 11), rat (10), dog (5) and pig (12). P53 consists 393 amino acids long and has three domains : (1) an $N$-terminal transcription-activation domain (TAD), which activates transcription factors, (2) a central DNA-binding core domain (DBD). Contains zinc molecules and arginine amino acid residues, and (3) a $C$ terminal homo-oligomerisation domain (OD). Tetramerization greatly increases the activity of P53 in vivo (Figure 1). 


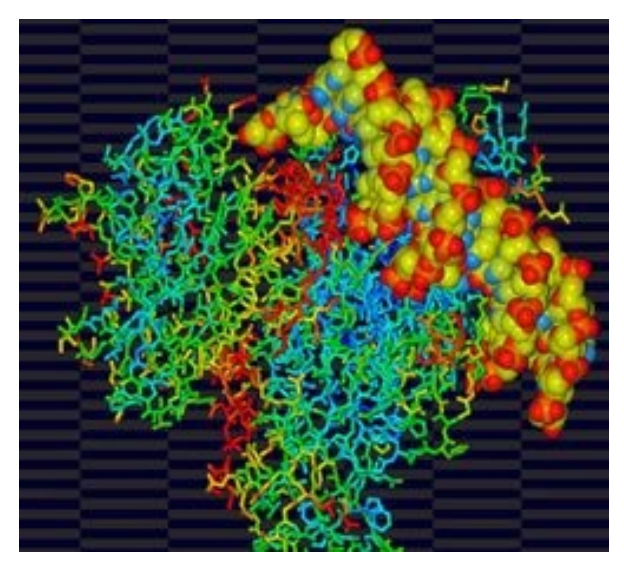

Figure 1. Representative figure of TP53 bound to a short DNA fragment. Protein atoms are represented as sticks, the DNA helix is in spacefill mode [8].

The $p 53$ gene is frequently mutated in human cancers and represents a good target for studying mutation spectra since there are $>100$ potential sites for phenotypic mutations. Most of the mutations that deactivate $p 53$ in cancer usually occur in the DBD which are recessive loss-of-function mutations. The mutations destroy the ability of the protein to bind to its target DNA sequences, and thus prevents transcriptional activation of these genes. Molecules of $p 53$ with mutations in the OD dimerise with wild-type $p 53$, and prevent them from activating transcription and have a dominant negative effect on the function of $p 53$. Wild-type P53 is a labile protein, comprising folded and unstructured regions which function in a synergistic manner [9]. Functionally, $p 53$ has many anti-cancer mechanisms. It can activate DNA repair proteins when DNA has sustained damage, it can also hold the cell cycle at the $G_{1} / S$ regulation point on DNA damage recognition and it can initiate apoptosis, the programmed cell death, if the DNA damage proves to be irrepairable.

Due to its highly important function, therefore, $p 53$ is central to many of the cell's anti-cancer mechanisms. As stated above, it can induce growth arrest, apoptosis and cell senescence. In normal cells $p 53$ is usually inactive, bound to the protein MDM2, which prevents its action and promotes its degradation by acting as ubiquitin ligase (Figure 2). Active $p 53$ is induced after the effects of various cancer-causing agents such as radiation, oncogenes and some DNA-damaging drugs. DNA damage is sensed by 'checkpoints' in a cell's cycle, and causes proteins such as ATM, CHK1 and CHK2 to phosphorylate $p 53$ at sites that are close to or within the MDM2binding region of the protein. Oncogenes also stimulate $p 53$ activation, mediated by the protein p14ARF. Some oncogenes can also stimulate the transcription of proteins which bind to MDM2 and inhibit its activity. Once 
activated $p 53$ transcribes several genes including one for $p 21$ that binds to the G1-S/Cdk and S/Cdk complexes (molecules important for the G1/S transition in the cell cycle) inhibiting their activity. p53 has many anticancer mechanisms, and plays a role in apoptosis, genetic stability, and inhibition of angiogenesis [8]. Recent research has also linked the p53 and RB1 pathways, via p14ARF, raising the possibility that the pathways may regulate each other [10].

In Figure 2 below, we can see that in A, MDM2 regulates P53 protein in three different ways. On binding to P53 transactivation domain, it inhibits its transcriptional activity (1), promotes P53 degradation (2), and favors the export of $p 53$ from the nucleus (3). Therefore, in the presence of MDM2, the P53 protein is inactivated and does not stimulate the expression of genes involved in apoptosis, cell cycle arrest, or DNA repair. In some tumors where MDM2 is overexpressed, P53 is constantly inhibited and tumor growth is favored. The inactivation of MDM2 in these tumors should activate the P53 pathway and as a possible consequence should activate apoptosis. And in B, several strategies can be used target to MDM2 in tumors. Antisense oligonucleotides should decrease the cellular levels of MDM2 (Strategy 1). Compounds that inhibit the ubiquitin ligase activity of MDM2 could prevent P53 degradation (Strategy 2). p14ARF acts by blocking MDM2-dependent degradation and transcriptional silencing of P53. p14ARF mimics should therefore activate the P53 pathway (Strategy 3). Inhibitors of the P53-MDM2 interaction should release P53 from MDM2 and as a consequence should activate protein P53 tumor suppressor activity (Strategy 4).
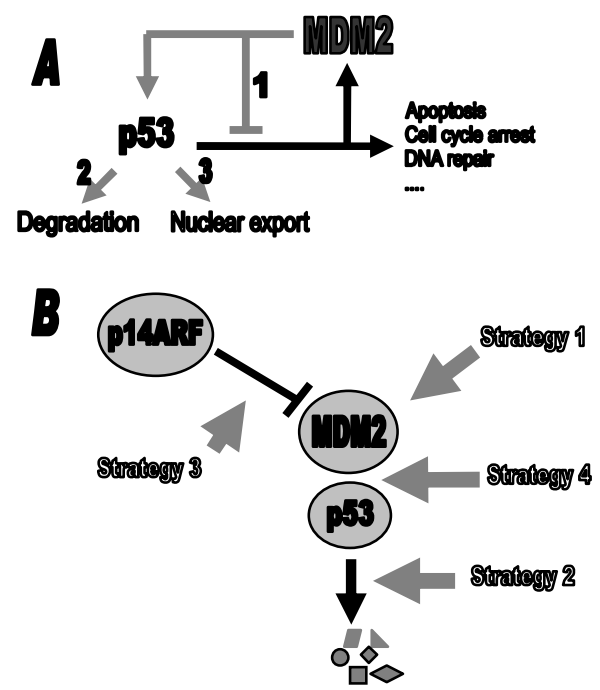

Figure 2. Regulation of protein p53 by MDM2 in three different ways (A) and several strategies used to target MDM2 in tumors (B). See text for detail mechanism [11]. 
If $p 53$ is damaged, tumor suppression is severely reduced. People who inherit only one functional copy of $p 53$ will most likely develop tumors in early adulthood, a disease known as Li-Fraumeni syndrome. Protein p53 can also be damaged in cells by mutagens, increasing the likelihood that the cell will begin uncontrolled division. Certain pathogens such as Human papillomavirus (HPV) can also affect $p 53$. HPV encodes for a protein, E6, which binds $p 53$ and inactivates it. This, in synergy with the inactivation of another cell cycle regulator, p105RB, allows for repeated cell division manifested in the clinical disease of warts. In health condition p53 is continually produced and degraded in the cell. The degradation of $p 53$ is associated with MDM2 binding. In a negative feedback loop MDM2 is itself induced by $p 53$. However $p 53$ mutant often don't induce MDM2, and are thus able to accumulate at very high concentrations. Mutant $p 53$ itself can inhibit normal $p 53$ [12].

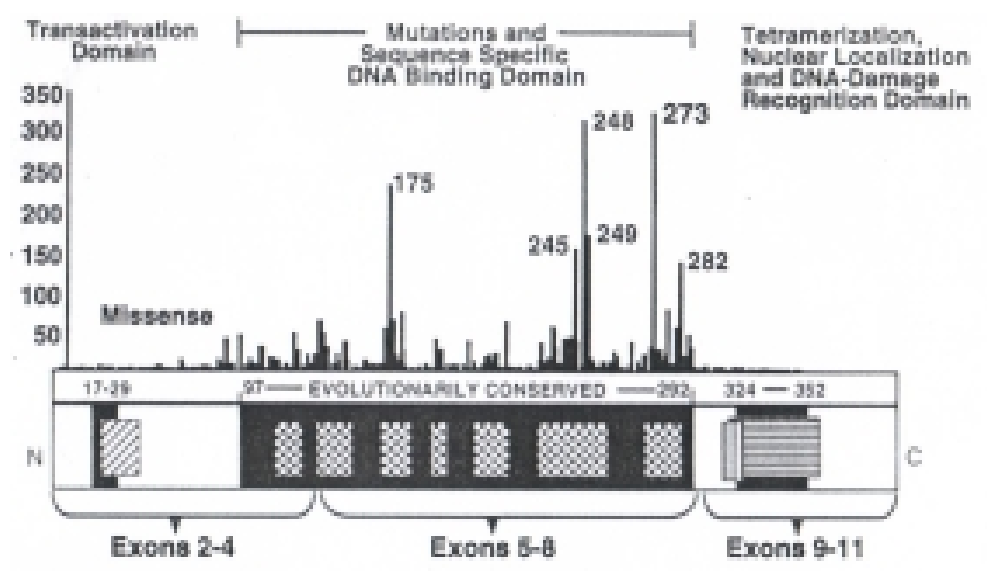

Figure 3. Representative of schematic of TP53 molecule showing the transactivation, evolutionarily conserved (mutation and DNA-bind), and tetramer and nuclear localization regions. Vertical bars show frequency of codons that mostly mutated (miss-sense) [7].

Since its initation of the first gene therapy clinical trial in 1990, more than 2100 patients world-wide have been entered into gene therapy trials for such diverse diseases as viral infection, monogenic defects ans disorders such as cancer. There are more than 71 clinical gene therapy trials in Europe, 60\% of which are in cancer. One of the current efforts focus on tumour supressor genes and manipulation of tumor growth and invasiveness, for example blocking angiogenesis. $p 53$ also has a potential therapeutic use. In-vitro introduction of $p 53$ in to $p 53$-deficient cells has been shown to cause rapid death of cancer cells or prevention of further division. It is these more acute effects which hopes rest upon therapeutically [13]. Restoring its function in cancer cells would be a major step in curing many cancers and various 
strategies have been proposed to do this [12]. A number of groups have found molecules which appear to restore proper tumour suppressor activity of $p 53$ in vitro by altering the conformation of $p 53$ mutant back to an active form. So far, no molecules have shown to induce biological responses, but some may be lead compounds for more biologically active agents. A promising target for anti-cancer drugs is the molecular chaperone Hsp90, which interacts with p53 in vivo. Importantly, normal p53 is exploited in radiation therapy.

As mentioned above that numerous antitumour therapeutic strategies have been proposed based on the ability to restore wild-type P53 function by refolding of mutant protein or relocalisation of mislocalised protein $[14,15]$. However, it is not yet clear whether continual $p 53$ inactivation is necessary for continued expression of the immortalisation phenotype, i.e. whether reexpression of wild-type levels of $p 53$ is sufficient to halt the proliferation of immortalised cells with different genetic backgrounds. This must be true for such therapeutic strategies to be successful. The ability to conditionally inhibit tumour suppressor function and subsequently restore wild-type levels of gene expression under normal physiological control is essential to answer this question and can also be used to examine functional relationships between genes in mammalian cells. While a variety of techniques are available to inhibit gene function, such as genetic disruptions in mice and somatic cell gene disruptions in human cultured cells, these techniques are effectively not reversible. An alternative approach which is applicable to a wide variety of cell types and is potentially reversible is the delivery or expression of antisense constructs.

\section{p53 STATUS AND IONIZING RADIATION}

Ionizing radiation induces genomic instability, which is transmitted through many generations after irradiation in the progeny of surviving cells $[16,17]$. Induced genomic instability manifests as the induction of various delayed phenotypes such as delayed lethal mutation or delayed reproductive death, delayed chromosomal instability, and delayed mutation induction [18]. Although genomic instability has been reported commonly in mammalian cells exposed to ionizing radiation, the mechanisms underlying the initiation and manifestation of radiation-induced genomic instability are not fully understood. Furthermore, other studies have suggested that an extranuclear target or a bystander effect may also be involved in the induction of instability [19]. Therefore, more than one mechanism may be involved in the initiation of radiation-induced genomic instability in survival cells. If the cell does not die, it may acquire genomic instability and lead to a population of cells with abnormally high susceptibility to gene and chromosomal instability, mutation and other delayed effects. Studies using inbred strains of rodents have clearly shown genotype-dependent differences in response to radiation 
exposure, including susceptibility to radiation-induced cellular transformation and tumor formation, as well as differences in susceptibility to radiationinduced chromosomal instability [20].

More specifically, ionizing radiation causes DNA double strand breaks, which are the initiators for reproductive death, chromosomal aberration, apoptosis, and mutation. Because all of these are manifestations of radiationinduced genomic instability, it is highly possible that delayed DNA damage is associated with delayed phenotypes. DNA double strand breaks are well known to accumulate and activate $p 53[21,22]$. Recent studies have described that DNA double strand breaks are recognized by ATM, and ATM-mediated phosphorylation of P53 protein accumulates and activates p53 [23]. It regulates transcription of the downstream genes such as $p 21^{\text {Wafl } / \text { Cipl } 1}$, gadd45, Reprimo, BAX, PIG-3, and p53AIP1 [7,24,25]. As a result, activated p53 causes cell cycle arrest, apoptosis, senescence-like growth arrest, and involved in base excision repair [26]. It was estimated that one single DNA double strand break is enough to activate $p 53$ [27], therefore, it is highly possible that DNA breaks arising in the progeny of surviving cells activate P53 protein. Moreover, radiation-induced genomic instability could be the driving force underlying the development of radiation-induced carcinogenesis by accumulating genetic alterations [28]. If $p 53$ function is a guardian of the genome, there should be delayed $p 53$ activation in the progeny of surviving cells [29].

It is well known from many studies that cells lacking normal p53 function exhibit no detectable $\mathrm{G}_{1}-\mathrm{S}$ arrest in response to radiation [30]. Following genotoxic damage, $p 53$ is induced and acts to restrain proliferation by inducing the expression of genes which lead to growth arrest (such as p21) or apoptosis (bax). And by inhibiting proliferation following DNA damage, p53 action prevents the accumulation of potentially oncogenic mutations. Loss of $p 53$ function has quite a varied effect on the radiosensitivity or chemosensitivity of tumor cells lines [31,32]. In primary mouse fibroblast cells which do not exhibit significant apoptosis in response to ionizing radiation, there is little to no preferential radioresistance of the $p 53$ null cells over the normal cells $[33,34]$.

$p 53$ also functions to suppress cellular immortalisation $[35,36]$. Normal primary somatic cells are capable of undergoing a finite number of divisions in culture until they undergo cellular senescence, characterised by growth arrest, a large flat morphology and insensitivity to further mitogenic stimulation [37]. In contrast, many tumour cells exhibit unlimited division potential, indicating that they have bypassed the barriers to immortalisation, such as cellular senescence. $p 53$ appears to play a direct role in controlling the onset of cellular senescence. $p 53$ transcriptional activity increases with ageing of the cells [38], wild-type $p 53$ activity is necessary for growth arrest in senescence and a high percentage of cells that escape from senescence have lost $p 53$ activity [39]. 
Persistent genomic instability or a mutator phenotype can result in the higher frequency of genetic alterations in cancer cells, which cannot be explained by random mutation [40]. Therefore, it has been hypothesized that radiation-induced genomic instability could be the driving force underlying radiation carcinogenesis $[41,42]$. However, some studies have shown that delayed DNA damage occurs in the progeny of surviving cells. Unscheduled DNA breaks may result in accumulation of genomic alterations. Because activated P53 protein in response to ionizing radiation has been thought to protect the integrity of the genome, delayed activation of P53 may also play a role as a guardian of the genome. The results of research by Clutton et al support that activated p53 causes delayed cell death including delayed apoptosis [43]. Therefore, radiation-induced genomic instability causes accumulation of genetic alterations, and delayed activation of $p 53$ plays an indispensable role in eliminating the damaged cells and maintaining genomic integrity. Although several studies have described the expression of delayed phenotypes, the molecular mechanisms involved in delayed induction of DNA damage have not been determined. It was proposed that potentially unstable chromosome regions resulting from DNA repair of double strand breaks are involved in delayed DNA breakage [44]. Previous studies demonstrated that dicentrics were the hallmark of chromosomal instability, and they provided a chance to cause DNA breakage during anaphase [45]. Although several mechanisms may be involved in the induction of delayed DNA breakage, it may be explained in part by the mechanism of manifestation of radiation-induced genomic instability. Additional studies are required to clarify the mechanism that causes delayed DNA breakage for prevention of accumulated genetic alterations in the progeny of surviving cells exposed to ionizing radiation.

Experimentally, numerous studies have shown that ionizing radiation can increase the frequency and decrease the age at which tumors are observed in mice. This observation is apparent in animals carrying mutations in known tumor suppressor genes and even more so in animals deficient in genes shown to have a role in DNA repair and maintenance of chromosome stability. For example, exposure of neonatal $p 53^{-/}$mice to ionizing radiation markedly decreases the latency to tumor formation. A similar decrease in tumor latency has been observed in irradiated $p 53^{+/}$animals [3]. This treatment also increased the frequency of malignant lymphomas and decreased the incidence of sarcomas in these animals. In contrast, exposure of $p 53^{+/}$mice to ionizing radiation did not alter the incidence of mammary tumors, and in fact no mammary tumors were identified on analysis of 33 treated $p 53^{+/}$animals [3]. These findings indicate that exposure to ionizing radiation can decrease tumor latency and alter the frequency with which specific types of tumors arise in p53-deficient animals. These results also suggest that differential exposure of humans and mice to such DNA-damaging agents alone cannot explain the extremely low incidence of mammary tumors in p53-deficient mice as 
compared to patients with the Li-Fraumeni syndrome. An additional explanation for the failure of $p 53$-deficient mice to develop mammary tumors may be the increasingly frequent finding that differences in the genetic background on which a tumor suppressor is studied can dramatically alter the tumor spectrum. It has been shown that the incidence of teratocarcinomas is elevated in $p 53^{-/}$mice on the $129 / \mathrm{Sv}$ genetic background as compared with $p 53^{-/}$mice on a mixed C57BL/6-129/Sv background. The role of $p 53$ in tumor suppression is further highlighted by the creation of $p 53^{-/-}$mice, which are highly cancer prone and develop a large spectrum of tumors. This suggests that different strains of mice contain sets of alleles that modify the impact of the loss of $p 53$ in a cell type-specific manner.

\section{TYPE OF $p 53$ MUTATION DEPENDENCE ON MUTAGEN}

The assessments on the influence of mutagen on the $p 53$ mutation prevalence and spectrum such as environmental tobacco smoke and residential radon for lung tumors, as well as ultra violet for the induction of skin cancer had been conducted by some researchers independently. Mutations in $p 53$ are the most common defects in lung cancer and may be a pathway through which environmental carcinogens initiate cancer. From investigation by Taylor et al [46] on the detection of $p 53$ mutations in lung cancers from uranium miners with high radon exposure was shown that 16 (31\%) of 52 large-cell and squamous-cell cancers from miners contained the same AGG to ATG transversion at codon 249, including cancers from 3 or 5 miners who had never smoked. This specific mutation has been reported in only 1 of 241 published $p 53$ mutations from lung cancers. They proposed that codon 249 mutation may be a marker for radon-induced lung cancer. Other examination conducted by Hollstein et al. [47] revealed that none of the lung tumors examined harbored the hotspot mutation. Five of the 50 tumors, however, did indeed harbor exon 7 mutations, as determined by subsequent mutation analysis of exon 7 . These mutations were dispersed among various codons and may be attributable to heavy tobacco smoking in this cohort. In support of this interpretation, they found no mutations in exons 5-8 of the $p 53$ gene in 13 iatrogenic liver cancers induced by injection of Thorotrast, an alpha-emitting radiocontrast agent. They propose that if the $p 53$ tumor suppressor gene is a target for the carcinogenic action of alpha-particle radiation, loss of suppressor function may occur preferentially by mechanisms such as intrachromosomal deletions, rather than by base substitution mutations.

Specific UV-induced mutation spectrum in the $p 53$ gene of skin tumors from DNA-repair-deficient xeroderma pigmentosum patients was found by Dumaz et al.[48]. All the mutations were located at dipyrimidine sites, essentially at CC sequences, which are hot spots for UV-induced DNA lesions. Sixty-one percent of these mutations were tandem CC-->TT 
mutations considered to be unique to UV-induced lesions; these mutations are not observed in internal human tumors. All the mutations, except two, must be due to translesion synthesis of unrepaired dipyrimidine lesions left on the nontranscribed strand. These results show the existence of preferential repair of UV lesions [either pyrimidine dimers or pyrimidine-pyrimidone (6-4) photoproducts] on the transcribed strand in human tissue. Literature suggests that the incidence of various tumors is determined largely by the genetic background on which mutations are studied. In addition, population studies and studies in animals suggest that environmental factors, together with genetic factors, determine overall risk for development of specific types of tumors. Environmental agents together with genetic factors can increase the frequency and decrease the latency of mammary tumors, leading to an incidence similar to that observed in Li-Fraumeni syndrome. Furthermore, it suggests that the risk of development of a particular type of tumor by individuals deficient in $p 53$ after exposure to damaging agents can be influenced by modifier alleles [49].

\section{SUMMARY}

The P53 protein has been implicated in multiple cellular responses related to DNA damage. When working properly, P53 binds DNA to activate genes that direct cells with damaged DNA to cease dividing until the damage can be repaired. Cells with such damage include cancer cells, since all cancers track to genetic flaws of one kind or another, whether inherited or acquired. If repairs cannot be made, P53 commands the cells with damaged DNA to self-destruct so they are no longer a danger to the body. Alterations in any of these cellular responses could be related to increased genomic instability. The $p 53$ gene regulates the G1 cell cycle checkpoint in response to DNA damage due to ionizing radiation. However, the significance of $p 53$ mutation in radiation sensitivity and its underlying mechanisms still remains unclear. To elucidate this case, some researches have measured the effects of p53 mutation not only on cell cycle delay, but also on apoptosis and radiation sensitivity using mouse cells transfected with different forms of $p 53$ mutations or human tissues. Other results revealed that cell survivals determined by clonogenic assay show that $p 53$ mutant cells are generally more sensitive to ionizing radiation than cells with wild-type $p 53$. These results suggest that mutant forms of $p 53$ represent a phenotype that affects the radiation sensitivity and is not dependent on the apoptotic pathway. These findings also provide the possibility that the observed instability results from these DNA breaks lead to delayed chromosome rearrangements, delayed cell death, and so forth, many generations after irradiation and that activation of $p 53$ function may eliminate cells that have potentially accumulated genomic alterations. In recent years, there has been an exponential increase in the number of p53 mutations identified in human cancers and 
its database consists of a list of point mutations in the $p 53$ gene of human tumors and cell lines.

\section{REFERENCES}

1. HARTWELL, L.H., HUNT, R.T. and NURSE, P.M., Key regulators of the cell cycle, Speech of Award of The Nobel Prize in Physiology or Medicine for 2001, Karolinska Institutet, 8 October (2001).

2. WILLIAM, G.T., Programmed cell death: apoptosis and oncogenesis, Cell, 65, 1097-1098 (1991).

3. LEE, J.M. and BERNSTEIN, A. p53 mutations increase resistance to ionizing radiation, Proceedings of the National Academy of Sciences, 90, 5742-5746 (1993).

4. LIN, A.W., BARRADAS, M., STONE, J.C., VAN AELST, L., SERRANO, M. and LOWE, S.W., Premature senescene involving p53 and $p 16$ is activated in response to constitutive MEK/MAPK mitogenic signaling, Genes Dev., 12, 3008-3019 (1998).

5. SERRANO, M., LIN, A.W., MCCURRACH, M.E., BEACH, D. and LOWE, S.W., Oncogenic ras provokes premature cell senescene associated with accumulation of p53 and pl6INK4a, Cell, 88, 593-602 (1997).

6. LAMBERT, B., Biological markers in exposed humans: gene mutations, in: Mechanisms of Carcinogenesis in Risk Identification (H. Vainio, PN. Magee, DB. McGregor et al., eds), IARC Scientific Publication 116 (1992).

7. VOGELSTEIN, B., LANE, D., LEVINE, A.J., Surfing the $p 53$ network, Nature, 408, 307-310 (2000).

8. KO, L.J. and PRIVES, C., p53: puzzle and paradigm, Genes. Dev., 10, 1054-1072 (1996).

9. BELL, S., KLEIN, C., MULLER, L., HANSEN, S., and BUCHNER, J., p53 contains large unstructured regions in its native state, $J$ Mol Biol., 322(5), 917-927 (2002).

10. BATES, S., PHILliPS, A.C., ClARK, P.A., STOTT, F., PETERS, G., LUDWIG, R.L., and VOUSDEN, K.H., p14ARF links the tumour suppressors $R B$ and $p 53$. Nature. 395, 124-125 (1998).

11. CHENE, P., Inhibition of the p53-MDM2 Interaction: Targeting a Protein-Protein Interface, Molecular Cancer Research, 2, 20-28 (2004).

12. BLAGOSKLONNY, M.V., P53: an ubiquitous target of anticancer drugs, Int J Cancer, 98(2), 161-166 (2002). 
13. MCCORMICK, F., Cancer gene therapy: fringe or cutting edge?, Nat Rev Cancer, 1, 130-141 (2001).

14. GALLAGHER, W.M. and BROWN,R., p53 oriented cancer therapies: current progress, Ann. Oncol., 10, 139-150 (1999).

15. KOMAROVA, E.A. and GUDKOV, A.V., Could $p 53$ be a target for therapeutic suppression?, Semin. Cancer Biol., 8, 389-400 (1998).

16. ORGAN, W. F., DAY, J. P., KAPLAN, M. I., MCGHEE, E. M., and LIMOLI, C. L., Genomic instability induced by ionizing radiation, Radiation Research, 146, 247-258 (1996).

17. LITTLE, J.B., Radiation-induced genomic instability, Int. J. Radiat. Biol., 74, 663-671 (1998).

18. SEYMOUR, C. B., MOTHERSILL, C., and ALPER, T., High yield of lethal mutations in somatic mammalian cells that survive ionizing radiation, Int. J. Radiat. Biol., 50, 167-169 (1986).

19. WATSON, G.E., LORIMORE, S.A., MACDONALD, D.A., and WRIGHT, E.G., Chromosomal instability in unirradiated cells induced in vivo by a bystander effect of ionizing radiation, Cancer Res., 60, 5608-5611 (2000).

20. WATSON, G.E., LORIMORE, S.A., CLUTTON, S.M., KADHIM, M.A. and WRIGHT, E.G., Genetic factors influencing alpha-particle induced chromosomal instability, Int. J. Radiat. Biol, 71, 535-555 (1996).

21. KASTAN, M. B., Signalling to $p 53$ : where does it all start?, BioEssays, 18, 617-619 (1996).

22. PRIVES, C., Signaling to p53: braking the MDM2-p53 circuit, Cell, 95, 5-8 (1998).

23. SUZUKI, K., KODAMA, S., and WATANABE, M., Recruitment of ATM protein to double strand DNA irradiated with ionizing radiation, $J$. Biol. Chem., 274, 25571-25575 (1999).

24. AGARWAL, M.L., TAYLOR, W.R., CHERNOV, M.V., CHERNOVA, O.B., and STARK, G.R., The p53 network, J. Biol. Chem., 273, 1-4 (1998).

25. VOUSDEN, K.H., p53: death star, Cell, 103, 691-694 (2000).

26. ZHOU, J., AHN, J., WILSON, S.H., and PRIVES, C., A role for $p 53$ in base excision repair, EMBO J., 15, 914-923 (2001).

27. HUANG, L-C., CLARKIN, K.C., and WAHL, G.M., Sensitivity and selectivity of the DNA damage sensor responsible for activating $p 53$ dependent $\mathrm{G}_{1}$ arrest, Proc. Natl. Acad. Sci. USA, 93, 4827-4832 (1996).

28. MENDONCA, M.S., TEMPLES, T.M., FARRINGTON, D.L., and BLOCH, C., Evidence for a role of delayed death and genomic instability 
in radiation-induced neoplastic transformation of human hybrid cells, Int. J. Radiat. Biol., 74, 755-764 (1998).

29. SUZUKI, K., YOKOYAMA, S., WASEDA, S., KODAMA, S. and WATANABE, M., Delayed reactivation of $p 53$ in the progeny of cells surviving ionizing radiation, Cancer Research, 63, 936-941 (2003).

30. KUERBITZ, S.J., PLUNKETT, B.S., WALSH, W.V. and KASTAN, M.B., Wil-type p53 is a cell cycle checkpoint determinant following irradiation, Proc. Natl. Acad. Sci. USA, 89, $7491-7495$ (1992).

31. MCILWRATH, A.J., VASEY, P.A., ROSS, G.M. and BROWN, R., Cell cycle arrest and radiosensitivity of human tumor cell lines: dependence on wild-type p53 for radiosensitivity, Cancer Res., 54, 3718-3722 (1994).

32. BRACHMAN, D.G., BECKETT, M., GRAVES, D., HARAF, D., VOKES, E. and WEICHSELBAUM, R.R., P53 mutation does not correlate with radiosensitivity in 24 head and neck cancer cell lines, Cancer Res., 53, 3667-3669 (1993).

33. SLICHENMYER, W.J., NELSON, W.G., SLEBOS, R.J. and KASTAN, M.B., Loss of a $p 53$-associated G1 checkpoint does not decrease cell survival following DNA damage, Cancer Res., 53, 4164-4168 (1993).

34. POWELL, S.N., DEFRANK, J.S., CONNELL, P., EOGAN, M., PREFFER, F., DOMBKOWSKI, D., TANG, W., and FRIND, S., Differential sensitivity of $p 53(-)$ and $p 53(+)$ cells to caffeine-induced radiosensitization and override of G2 delay, Cancer Res., 55, 1643-1648 (1995).

35. FOSTER, B.A., COFFEY, H.A., MORIN, M.J. and RASTINEJAD, F., Pharmacological rescue of mutant p53 conformation and function, Science, 286, 2507-2510 (1999).

36. GAO, Q., HAUSER, S.H., LIU, X.L., WAZER, D.E., MADOC-JONES, $\mathrm{H}$. and BAND, V., Mutant p53-induced immortalization of primary human mammary epithelial cells, Cancer Res., 56, 3129-3133 (1996).

37. BARRETT, J.C., ANNAB, L.A. and FUTREAL, P.A., Genetic and molecular basis for cellular senescence, Adv. Exp. Med. Biol., 330, 27-43 (1993).

38. BOND, J.A., HAUGHTON, M., BLAYDES, J., GIRE, V., WYNFORDTHOMAS, D. and WYLLIE, F., Evidence that transcriptional activation by $p 53$ plays a direct role in the induction of cellular senescene, Oncogene, 13, 2097-2104 (1996).

39. DALAL, S., GAO, Q., ANDROPHY, E.J. and BAND, V., Mutational analysis of HPV type 16 E6 demonstrates that $p 53$ degradation is necessary for immortalization of mammary epithelial cells, J. Virol., 70, 683-688 (1996). 
40. LOEB, L.A., Mutator phenotype may be required for multistage carcinogenesis, Cancer Res., 51, 3075-3079 (1991).

41. MENDONCA, M.S., TEMPLES, T.M., FARRINGTON, D.L., and BLOCH, C., Evidence for a role of delayed death and genomic instability in radiation-induced neoplastic transformation of human hybrid cells, Int. J. Radiat. Biol., 74, 755-764 (1998).

42. ULLRICH, R.L. and PONNAIYA, B., Radiation-induced instability and its relation to radiation carcinogenesis, Int. J. Radiat. Biol., 74, 747-754 (1998).

43. CLUTTON, S.M., TOWNSEND, K.M., WALKER, C., ANSELL J.D., WRIGHT E.G., Radiation-induced genomic instability and persisting oxidative stress in primary bone marrow cultures, Carcinogenesis (Lond.), 17, 1633-1639 (1996).

44. SUZUKI, K., Multistep nature of X-ray-induced neoplastic transformation in mammalian cells: Genetic alterations and instability, $J$. Radiat. Res., 38, 55-63 (1997).

45. MARDER, B.A., and MORGAN, W.F., Delayed chromosomal instability induced by DNA damage, Mol. Cell. Biol., 13, 6667-6677 (1993).

46. TAYLOR, J.A., WATSON, M.A., DEVEREUX, T.R., MICHELS, R.Y., SACCOMANNO, G., and ANDERSON, M., P53 mutation hotspot in radon-asociated lung cancer, Lancet, 343, 86-87 (1994).

47. HOLLSTEIN, M., BARTSCH, H., WESCH, H., KURE, E.H., MUSTONEN, R., MUHLBAUER, K.R., SPIETHOFF, A., WEGENER, K., WIETHEGE, T., and MULLER, K.M., $p 53$ gene mutation analysis in tumors of patients exposed to alpha-particles, Carcinogenesis, 18, 511-516 (1997).

48. DUMAZ, N., DROUGARD, C., SARASIN, A. and DAYA-GROSJEAN, L., Specific UV-induced mutation spectrum in the $p 53$ gene of skin tumors from DNA-repair-deficient xeroderma pigmentosum patients, Proc Natl Acad Sci USA, 90, 10529-10533 (1993).

49. BACKLUND, M.G., TRASTI, S.L., BACKLUND, D.C., CRESSMAN, V.L., GODFREY, V. and KOLLER, B.H., Impact of Ionizing Radiation and Genetic Background on Mammary Tumorigenesis in p53-deficient Mice, Cancer Research, 61, 6577-6582 (2001). 